\title{
Research on the Accurate Continuous Calibration System for Current Transformer
}

\author{
Yonghong Fan ${ }^{\mathrm{a}}$, Jiandong Zhao and Huaguang Li \\ ${ }^{1}$ Qingdao Huanghai University, Qingdao, Shandong, China \\ a fanyonghong_618@163.com
}

Keywords: Current Transformer, Accurate Continuous, Calibration System

\begin{abstract}
The current transformer, as one of the basic electrical system components, by its accuracy and stability, ensures the metering, protection and analysis of the power system. The current transformers are wildly used since 1884, when the electromagnetic transforms were invented. Recently, the capacity of electrical power is greatly enhanced, and the intelligent electric grid is built. To meet the requirement of intelligent electric grid, the electronic current transformer is substituting for the conventional current transformer because of it is without a ferromagnetic core and it has the wide dynamic range and the frequency band.
\end{abstract}

\section{Introduction}

Power system, the role of transformer in the case to achieve a high voltage side and the secondary side equipment, electrical isolation, and secondary equipment to ensure personal safety related electrical equipment and power system parameters were measured in order to provide measurement, monitoring and protection basis to security systems, economic operation. In the power system during normal operation, the current transformer can measure current information on the primary side, the secondary side output to measuring and metering means for measuring current and power systems. When the power system failure, the current transformer can accurately reflect the current waveform under fault conditions, and with protection with the power grid failures constitute various protection and automatic control, and therefore plays a transformer in the safe operation of the power system a very important role.

At present, China Power System Application Current transformers are mostly traditional electromagnetic current transformer, the output signal is a current signal (5A or $1 \mathrm{~A}$ ), published since 1884 transformers, electromagnetic power transmission equipment has been fully developed, wherein the electromagnetic current sensor is one of the most important applications. But with the development of power industry, the power transmission system power capacity increasing power grid voltage level higher and higher. The voltage level of increase, the safety performance of the grid with new challenges, especially as a basis for the sensing unit transformers are increasingly high requirements. With the digital grid in the promotion of our country as a digital grid of the most important primary equipment - electronic transformer has been widespread concern. Traditional electromagnetic current transformer principle of the existence of defects, such as complex insulation structure, the inherent magnetic saturation and smart grid output does not meet the requirements, will gradually be replaced by electronic transformer.

Any kind of metering equipment must be regularly inspected, and electronic transformer due to the new features of its signal conversion processes and the corresponding reforms at home contains a lot of electronic components, on-site verification of its norms and traditions transformer compared to needs. Periodic intervals up to 10 years cannot be verified, it should be subjected to verification standards for electronic energy meter, greatly reducing the periodic on-site calibration intervals to prevent early failure of the transformer in electronic components and bring to the grid the blackouts. Since the electronic transformer is a new beginning toward practical technology, it is currently the object of study is less check technology, particularly its research site inspection techniques live almost entirely empty. Online verification of digital electronic transformer technical specifications can be found in the premise of ensuring the reliability of power supply, provide an effective means 
of long-running electronic transformer performance testing, it is in line with the overall requirements of network development, the digital grid the healthy development of great significance, has a very good prospect.

\section{The Classification and Basic Principles of Current Transformer}

Electromagnetic Current Transformer. The traditional current transformer is based on the principle of electromagnetic induction type transformer. Its primary winding connected in series in the power circuit, the external circuit to the secondary winding of measuring instruments, energy measurement and protection devices. Electromagnetic current transformer works shown in Figure 1, and its structure is similar to a transformer, around which a secondary winding on the core, relying on an electromagnetic coupling between the secondary winding of the current value from the current loop power system primary rotor into small secondary side current output.

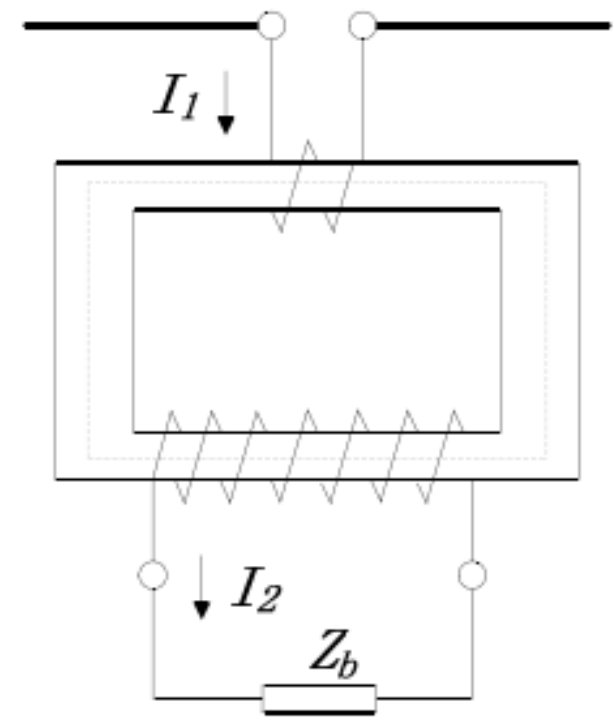

Fig. 1 The works principles of electromagnetic current transformer

Optical Current Transformer. Optical current transformer (OCT), also known as passive type current transformer, there are all-fiber magneto-optic current transformers and current transformers, the use of the light intensity, changes the polarization state, wavelength and measuring current. In the magneto-optical current transformer for example. Using optical measurement principle and transmitting digital signals using optical fiber, it is one of the important development of electronic Transformer. The principle is Faraday effect, that linearly polarized light passes through

\section{The Main Problems of the Current Transformer Calibration System}

The existing parity principle is needed to improve. With the promotion of digital substation, electronic transformer applications, from the principle of parity, the traditional calibration device cannot meet the new needs. Development of new calibration device has become a new hot spot verification industry research. Especially for digital output electronic current transformer, because it requires a digital interface, error test is more complex than the traditional transformer secondary output. Traditional calibration device from the interface device to check principle requires a corresponding improvement.

The existing off-line regular preventive check mode needs to be improved. From the calibration method, the current application of the current transformer calibrator mostly applied only to production test and field test transformer products offline or regular maintenance. Require periodic tests and overhaul outage, it will cause great economic losses, and human resources. Regular preventive test conditions differ from the offline operating condition, most trials are under low-voltage electromagnetic interference situation, so may not detect potential defects and malfunctions. 
Put into operation in the electronic current transformer calibration cycle is too long. Traditional transformers only analog current or voltage signal, only the traditional outdoor transformer core and coil of the passive, high reliability of the conventional transformer. Therefore, the existing current transformer regular maintenance needs of electromagnetic current regularly check for 5 years or 10 years is in line with its law of development. In the digital substation, since the electronic transformer increasing the signal conditioning and conversion circuit, so that a large number of electronic components in the outdoor run, the stability of the electronic current transformer is not as traditional current transformers. For electronic current transformer intervals regular check up to 10 years cannot be shortened periodic on-site calibration intervals to prevent early failure in transformers and electronic components to the power grid caused by large area blackouts.

The existing on-site verification systems still require up-flow devices. Existing so-called on-site verification system, only in the laboratory with a portable treatment based on current transformer calibration system, in field testing and it still need power to operate and up-flow. Power system voltage level continues to improve, up-flow device requirements needed to check on-site testing, high voltage insulation and electromagnetic interference and other issues are also increasingly serious, not only will lead to deterioration of insulation caused by the electromagnetic field, as well as mechanical force or heat , may eventually lead to insulation failure.

The existing verification system does not apply to standard pickup line verification system. Since the high-power power electronic devices to cause some degree of power system harmonics, inter-harmonics pollution, so the traditional transformer as a standard sensor head band-limited, cannot fully meet the needs of measurement accuracy.

\section{The Basic Theory of Online Verification System}

Online verification system includes standard channel, the channel be corrected and verified platform of three parts and the basic principle is: the standard current sensor and the correction of the current sensor output is sent to check the platform were measured through standard channels and channel. Verification platform has two output signals of the same timing signal then the error-calculated amplitude and phase values of the two signals are obtained, more than the difference between the results and phase errors. The online verification system that compared with laboratory calibration system differences: 1) standard current sensor designs. Laboratory standard current sensor with high accuracy multi electromagnetic current transformer, the system uses a clamp for easy on-site installation based on hollow coil PCB board as the standard sensor head. 2) Error Theory algorithm. Absolute comparative measurement principle of the need for accurate calibration system that extracts a sample value at a time of two wire current, namely, the standard current transformer current transformer output value and measured output value. Online harsh electromagnetic environment calibration system working, compared with laboratory calibration system, the need to improve the error theory algorithms, more precisely extract the two signals at the fundamental frequency parameter value in order to do a comparison operation.

\section{The Configuration of Online Verification System}

Electromagnetic current transformer online calibration system consists of standard channels, and verified by the school channel platform of three parts. It is corrected by the school channel including transformer output I / V converters and low-side signal acquisition module. Standard sensors based on hollow coil clamp PCB board. High side signal acquisition modules include pre-amplification, analog to digital conversion and electro-optical conversion, after receiving the synchronization signal hollow coil output differential signal preamplifier, A / D conversion, and then sent via fiber optic module to send data to local receiver module. Measured by the current transformer I / V converter, the output current signal is converted into a voltage $0 \sim 5 \mathrm{~A}$ signal to the low-pressure side signal acquisition module. The low pressure side of the main acquisition module includes analog to digital conversion and electro-optical conversion, after receiving the synchronization signal I / V converter output voltage signal samples is converted to an optical signal 
is transmitted to the local receiver module. Local receiver module includes a clock on the photoelectric conversion and laser power. Two-way module sends a synchronization signal, the data signal acquisition module for high side can send and receive two channels back to RS-232 communication interface standard is sent to the host computer platform virtual instrument calibration complete signal processing and computing error diagnosis.

\section{The Key Technology of Online Verification System}

The Standard Sensor Head Design. Online verification of the need for the system to achieve the goal-line uninterruptible power monitoring, must meet the standard sensor head simple structure, reliable, easy to install, can be implemented on-site installation in the case of primary current uninterruptible power. Current power system metering current transformer universal level of 0.2, 0.5 and special purpose $0.2 \mathrm{~s}$, $0.5 \mathrm{~s}$ level, measured according to the requirements of the standard must be higher than the measured sensor accuracy sensor accuracy level above two, namely 0.05. UPS To achieve the primary current run, standard sensors only use clamp structure or a non-contact measuring current sensing head, the current non-contact measurement of current sensing head from the principles and processes cannot meet the requirements of high accuracy and it cannot meet the standards required accuracy of the sensor head. Therefore, only select clamp sensor head.

High Accuracy Data Processing Algorithm. Whether it is the current standard test sensor head or sensor head out of the induced voltage signal is ultimately after A / D sampled into discrete digital signals into the host computer for calculations, the PC software required for data processing by samples, calculate the RMS and other electrical parameters of the initial phase information to obtain ratio and phase difference error results. Fourier Transform error calculation method is one of the most widely used electrical parameter estimation method. Discrete Fourier transform (Discrete Fourier Transform, DFT) is the Fourier transform of a discrete time domain. It can be time-series discrete spectral analysis and calculation, and the result is converted to the frequency domain finite length discrete sequence of signals and analyzed by a computer system it provides a very powerful tool. DFT as a common power system parameter measurement and estimation methods, laboratory extraction is the most common fundamental frequency parameter calculation algorithm error of current transformer calibration system.

\section{Conclusions}

This article describes the overall configuration and the key technology of the online verification system, given the basic structure of the online verification system for test subjects were divided into electromagnetic current transformer online verification system, the analog output electronic current transformer online check system and digital output electronic current transformers online verification system. Including standard and high-precision sensor head design error theory studies algorithms.

\section{References}

[1] Zhenya Liu: Journal of State Grid, Vol. 6 (2004) No 53, p.25-26

[2] Jianbo Guo: Power System Technology, Vol. 12 (2005) No 27, p.74-76

[3] Guinxin zhang: Electric Equipment, Vol. 1 (2006) No 33, p.11-14

[4] Jieming Liu: Huazhong University of Science and Technology, Vol. 3 (2007) No33, p.121-124

[5] Bo Ning: Power System Protection and Control System, Vol. 1 (2006) No 33, p.11-14

Fan Yonghong (1982-), female, she is now a lecture in the Qingdao Huanghai University. Her main research interests are electronic and communications. Tel: 15020028872 